\title{
Coral Reef Image Classifications with Hybrid Methods
}

\author{
C. Padma Priya, S. Muruganantham
}

\begin{abstract}
There are several organisms on oceans. Among the organisms coral reefs are the one with 800 species. Classifying coral is a difficult task. Scientist classify the coral organism and put in to groups based on their characteristics. There are several machine learning algorithms are implemented to analyzer and classify the coral species. The main aim of this work is to effectively use handcrafted features with deep features for classifying the coral classes. Here the state of art feature descriptors such as Local Binary Pattern, Local Arc Pattern and Improved Webbers Binary Code are proposed to extract the features of coral. The results which obtained can be further improved by combining these local descriptors with convolution neural network. The feature extracted by above methods are classified using KNN and Random Forest. Experiments with these methods are conducted using EILAT dataset. The Experimental results obtained by these methods demonstrate the effectiveness and robustness of our proposed method.
\end{abstract}

Keywords: LBP,KNN,RandomForest, Local Arc Pattern, Improved Webers Binary code.

\section{INTRODUCTION}

The Coral reefs are nautical invertebrate animals made of calcium carbonate. Coral reefs are one of the most miscellaneous ecosystem goods and services they afford to marine tropical and subtropical nations. Coral reefs provide shelter to various species living in seawater. Coral reef play an important role in coastal zone which it acts as a natural breakwater to minimize wave impacts from storms. Human effect on coral reefs is extensive. Coral reefs have risen world-wide prominence in terms of their quantities to act as early cautiousness for universal climatic variation. Death of coral reefs is caused by the environmental changes, pollution and increase of water temperature [1],[2].Plastic Pollution is one of the factor which puts worlds coral reef at risk. The examination of underwater images is an rousing computer vision problem. Some of the monitoring techniques which are used are Roving Diver Survey,Quadrat,Transectlines, the Manta tow. Coral reef are three dimensional images that look differently at different view point and Scaled Analysis of coral images which was conducted manually, was both time

Revised Manuscript Received on September 22, 2019.

C.Padma Priya, Research Scholar in Computer Science (18223152162024) ST.HinduCollege,Nagercoil, Affliated to Manonmanium Sundaranar University , Tirunelveli-627412.

Dr.S.Muruganantham, Associate Professor of Computer Science - S T.HinduCollege,Nagercoil, Affliated to Manonmanium Sundaranar University ,Tirunelveli-627412.

consuming and prone to error. There are inherent difficulties in using classification methods for analyzer the coral reef due to water turbidity which affect ambient light and image color. Imaged classification could be complex and challenging task. There are numerous classification methods used. This chapter deliberates latest research in classifying coral reef using CNN embedded with handcrafted features such as Local Arc Pattern, Local Binary Pattern and Improved Webers

\section{A. Binary Code Descriptor}

\section{The contribution of this work are the following:}

- Analysing and classifying the under water coral reefimages using handcrafted features.

- Discussing the layers of CNN and its structure.

- Exploring the results based on implementation of CNN embedded with Local Binary Pattern, Pattern,Local Arc Pattern and Improved Webers Binary CodeDescriptor seperately and analysing the results.

- Comparing the results.

\section{RELATED WORK}

Image classification is an necessary step in detection and study of coral reefs. A lot of image classification systems have been predicted till date. Satellites are the wonderful device for spotting the Earth and Ocean. It is used to classify the species of the coral reef. Several classification technology are implemented to suggest the best classification methods which gives better accuracy in classifying the species of coral.In this study various classification methods are observed by its results and accuracy which was implemented by various authors. Marcos [3] applied a feed forward back propagation neural network to depict Coral reef in to three benthic classifications living coral, dead coral and sand. Regression and classification problems are solved using Decision-tree algorithms.The features of corals are depicted using Local Binary Patterns (LBP). 


\section{Coral Reef Image Classifications with Hybrid Methods}

Paul Anton Letnes [4] used multi or hyper spectral imagery from satellite and aeroplanes to analyse coral.Machine learning method which is used here is used to classify the large scale image classification of coral species.SVM classification algorithm and Scikit-learn software packages for PLS areused.Mehta[5] implemented Support Vector Machine (SVM)for analysing the texture of coral reef for better performance.Radial Basis kernel function is alsoused.Pizarro [6] presents a preliminary investigation in using a state-of-art bag of features for object recognition system to classify coral based on labeled examples.

M.D.Stokes and G.B.deane[7] used normalized color space and discrete cosine transform in a statistical distance based classification for classifying the coral images which was collected by ecologist.[8] classify coral species using Local Binary pattern(LBP) and CLBP method.Shihavuddin[9] implemented K nearest neighbour (KNN),probability density weighted mean distance(PDWMD),support vector machine (SVM),neural network(NN).Nurhalis[10]objective was to implement Support Vector Machine(SVM),Random Tree, Decision Tree(DT),Bayesian and k-nearest neighbour(KNN) to achieve accuracy of coral reef benthic habitat mapping classifications. Mohamed [11] proposed CNN which is a supervised deep learning method used for coral classification. Mahmood [12] proposed VGG Net for object recognition which is an convolutional neural network. Theembedded CNN based features with hand crafted features are used for better classification performance.[13] implemented convolutional neural network for better classification and recognition tasks.

Jean-Nicola Blanche [14] presented VGG Net for classification problems. Neural network and nearest neighbour classifiers are used.YANGuoqiang [15] implemented Support Vector Machine (SVM) to monitor the healths of coral reefs.M.Bennamoun[16] presented Resfeats for image classification and object detection. The deep layers of $\mathrm{CNN}$ is used to give best performance in classification.AniBrownMary [17] proposed Improved Local Derivative Pattern (ILDP) for classification. It achieves highest accuracy compared to other classification methods.InigoAlonso[18] presented CNN models for semantic segmentation.Superpixels SLIC-GT, SEEDS-GT implemented for better segmentation.LianXu [19] proposed CNN and VLAD for analysing and classifying the coral.The embedded features of FC and CONV are used in coral image classification.Dianpeng [20] proposed Support Vector Machine for classification. Anabel [21] proposed CNN with Inception V3, ResNet, and Dense Net for classifying coral species. DataAugumentation techniques, CNN architecture, and transfer learnings are examined. gawa [22] proposed Hyper spectral BI imagery and SVM regression model for representing the bottom types of coralreefs.[23] proposed a deep learning method to evaluate the population of coral. To classify coral from non coral Resfeats,convolutional neural network are utilized.[24] proposed Z with Tilted Z Local Binary Pattern (ZTZLBP) for coral reef imageextraction.KNN is used for image classification.

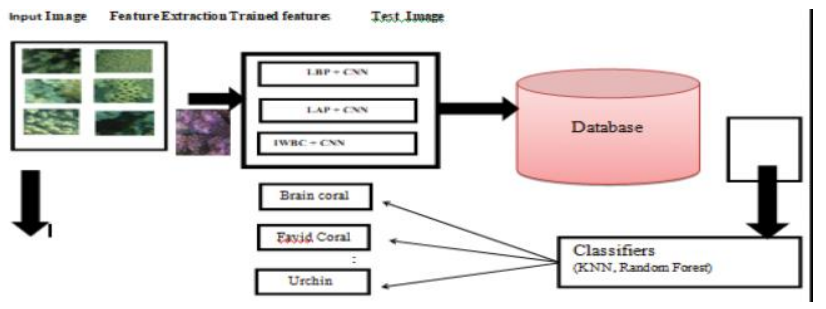

\section{METHODOLOGY}

The methodology implemented the state of art deep learning method to classify the coral reef classes.Figure lextant the cascaded approach for coral classification that intellectually combine the CNN model and handcrafted features.The commonly used handcrafted features include various morphological, shape, statistical, and textural features that endeavor to model the appearance of the domain and in particular the appearance of the coral with in digitized images. A recent major development in image classification has been the use of deep convolutional neural networks (CNNs), with excellent results. The Test images of coral which are classified using the classifiers such as KNN and Random Forest. The classified results shows the various classes of coral.

The methodology which are implemented in the proposed are:

- Deep Features of Convolutional Neural Network(CNN).

- The Handcrafted Features such as

- Local Binary Pattern(LBP)

- Local Arc Pattern (LAP)

- ImprovedWebers Binary Code(IWBC)

\section{A. Convolutional Neural Network}

Cnn is a specialized type of neural network used sprusely for image recognition and classification.CNN are a supervised learning method and are trained using data labeled with the respective classes.It is built up of neurons.Each neuron accepts several inputs,take weighted sum and sent it through activation function and respond with an 
output.CNN image classifications ensues an input image,process it and classify it under categories. Convolutional neural network which is used in the proposed method is used to classify coral reef images. It is the first layer to extract features from an input image. It use filters to detect the presence of specific features or patterns present in the input image. The size of the input image which is used in the proposed system are $(64 \times 64 \times 3)$, where 64 is width,next 64 is height and 3 represent RGB channel values. Convolution conserves the relationship between pixels by learning image features using small squares of input data.image matrix and a filter or kernel are taken as two inputs for its mathematical operations. This filter is lap across the width and height of the input file, and a dot product is enumerated to give an activation map.This proposed method consist of three convolutional layers. TheReLu Layer is added after each convolutional layer.TheRelu layer is the activation layer which specify all negative pixels to zero and transact element wise operation. It precedes non-linearity to the network. The output is the recalibrate feature map. The next layer which is followed by Relu layer is the maxpooling layer here in the proposed method two maxpooling layer is used.Next is the fully connected layer which is necessary to attach.It takes output from these convolutional neural network results

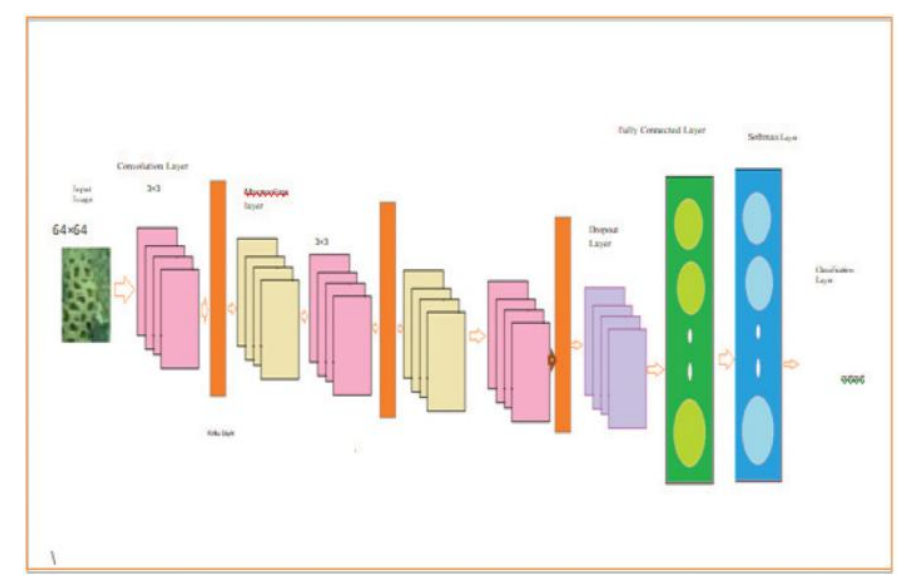

Figure 2: Proposed Cnn Architecture

in a $\mathrm{N}$ dimentionalvectors. Where $\mathrm{N}$ represent the numberof classes.After these layer follows dropout layer which is used to prevent overfitting in the proposed system.finally these layers are followed by softmaxlayer.The classification layer gives the desired output. This method is used to extract deep features trained on Eilatdataset.This method provide auspicious performance for different classification.

\section{B. Local Binary Pattern (LBP)}

Local Binary Pattern is a type of image descriptors used for classification in computer vision.It is a prevailing feature used in the proposed method for texture classification. The
Local Binary operator works in a $3 \times 3$ pixel of coral reef image

\begin{tabular}{|l|l|l|}
\hline 5 & 9 & 1 \\
\hline 4 & 4 & 6 \\
\hline 7 & 2 & 3 \\
\hline
\end{tabular}
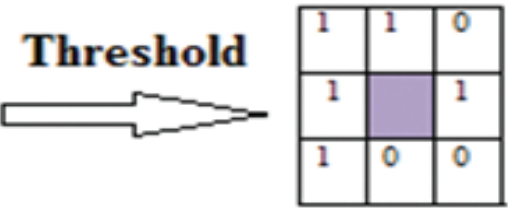

Figure 3: An Example for basic LBP operator

Binary: 11010011

Decimal:211

It is one of the image operator which recast an image in to an array or image of integer labels.It outline the textures of the image.The LBP operator replaces the value of the pixels of an image with decimal numbers, which are called LBP.Each central pixel is collated with its eight neighbourhood pixel.If the center pixel value is larger or identical to its neighbourhood pixel then the value of the bit is 1 and if the center pixel is lesser than its neighbourhood pixel then the value of the bit is 0.For each given central pixel one can produce a binary number which is obtained by linking all these binary bits in a clockwise manner which starts from the one of its top-left neighbour.The resulting decimal value of the generated binary number displace the central pixel value. The histogram of LBP intended over the image or region of the proposed system can used as texture descriptor to coral images.

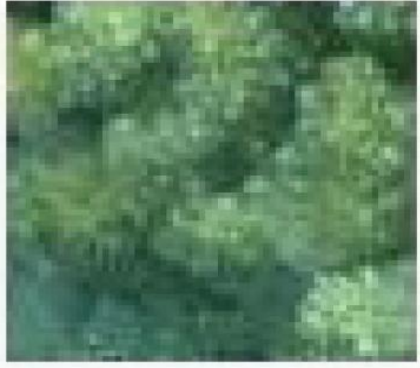

(a)

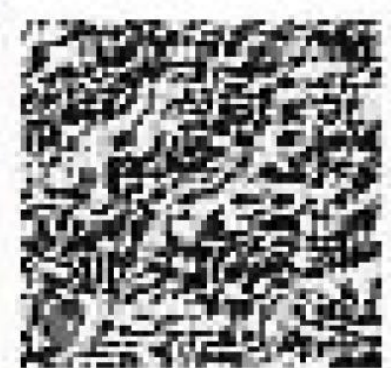

(b)
Figure 4: a)Original Image of Coral b) Image after LBP operation 


\section{Coral Reef Image Classifications with Hybrid Methods}

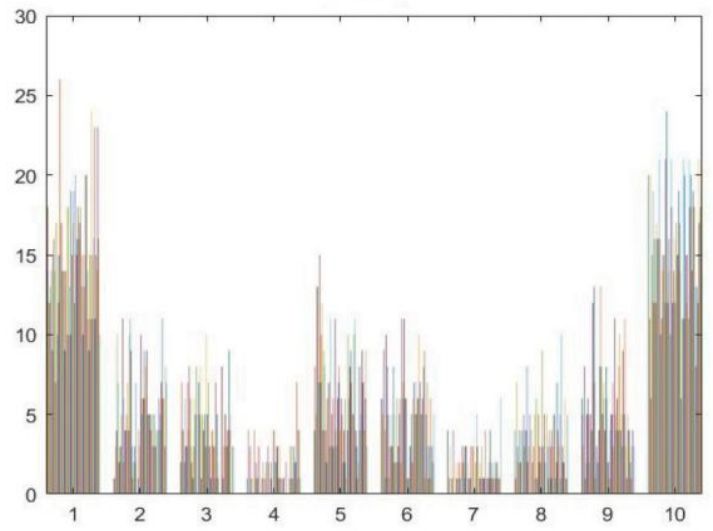

Figure 5: Histogram of LBP over coral image

\section{Local Arc Pattern (LAP)}

Local Arc Pattern is a Local feature descriptor for coral image recognition.Local Arc Pattern of center pixel is calculated using $5 \times 5$ pixels.LAP consist of Pattern-1(P1) with 4 bit binary pattern and Pattern-2(P2) with 8 bit binary pattern.The pattern 11 have 16 bit combination and pattern p2 have 256 bit combination.A bin is created for each combination to count the number of occurences of the combination within a given block.P1 will generate sixteen bins and $\mathrm{p} 2$ will generate 256 bins. $\mathrm{P} 1$ and $\mathrm{p} 2$ are concatenated to build LAP histogram for a block. The feature vector length per block will be 272 by adding 16 and 256.The final feature vector can be calculated by concatenating the histograms of all blocks of an image.The coral image which are given in this proposed are operated with LAP and the histogram also obtained which are listed in Figure 8.

\begin{tabular}{|l|l|l|l|l|}
\hline bl & b2 & b3 & b4 & cl \\
\hline c8 & al & a2 & a3 & c2 \\
\hline c7 & a8 & & a4 & c3 \\
\hline c6 & a7 & a6 & a5 & c4 \\
\hline c5 & b8 & b7 & b6 & b5 \\
\hline
\end{tabular}

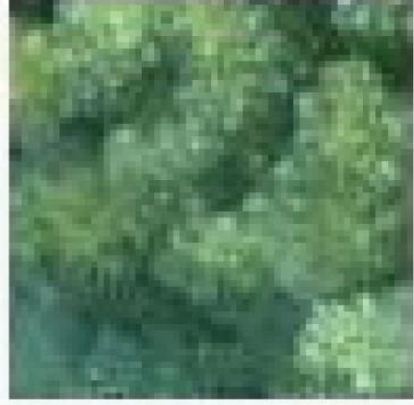

(a)

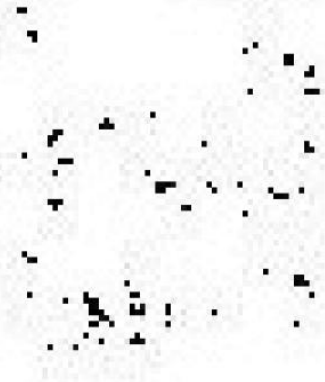

(b)
Figure 7 :a)Original image of coral (b)Image after LAP operation

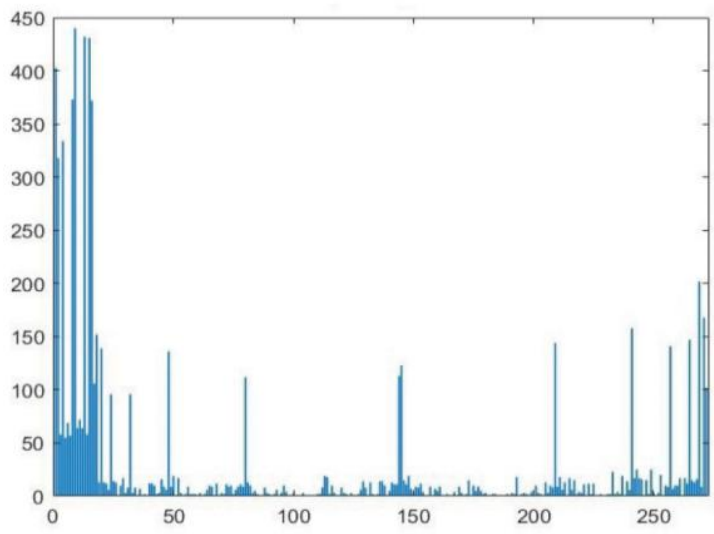

Figure 8: Histogram of LAP operated coral image

\section{Improved Webers Binary Code(IWBC)}

Improved Weber Binary Code Descriptor are derived from Webers Lawwhich encodes IWLD features of novel Weber magnitude and Weber orientation.The Local Binary Magnitude Pattern(LBMP) are obtained by encoding novel weber magnitude with the traditional methods of Local Binary Pattern(LBP).Similarly the Local XOR Orientation are obtained by encoding novel weber orientation using Local XOR pattern(LXP)[25] Binary pattern [,] of novel magnitude and orientation embedded in IWLD are obtained by following rule:

After performing encoding of IWLD we obtained Improved Weber Binary code(IWBC) map.The code maps of Weber magnitude and orientation are represented by IWBC-M and IWBC-O respectively.IWBC-M and IWBC at each location are obtained as follows:

$$
\begin{gathered}
B_{l}^{\xi}\left(k_{c}\right)=[0 \text { if } s \mathrm{~m}-1>0 \\
1 \in\{\mathrm{b}\}(1) \\
1 \text { if }\{\xi \mathrm{m}-1 \leq 0
\end{gathered}
$$

Figure 6: Sample 5×5 pixels with Pattern 1 and Pattern 2 


$$
\operatorname{IWBC}-\mathrm{M}\left(k_{c}\right)=\left[B_{a}^{\hat{t}}\left(k_{c}\right), B_{b}^{\tilde{\xi}}\left(k_{c}\right), \ldots \ldots \operatorname{LBMP}\left(k_{c}\right)\right]
$$

Binary (2)

$\begin{array}{ll}\begin{array}{l}\text { Orientation } \\ \text { Quantization }\end{array} & \begin{array}{l}\text { Binary } \\ \text { Quantizatio }\end{array} \\ & \end{array}$

\begin{tabular}{|l|l|l|}
\hline 76 & 184 & 189 \\
\hline 134 & 87 & 86 \\
\hline 315 & 210 & 42 \\
\hline
\end{tabular}$\longrightarrow$\begin{tabular}{|l|l|l|}
0 & 2 & 2 \\
\hline 1 & 0 & 0 \\
\hline 3 & 2 & 0 \\
\hline
\end{tabular}$\quad$\begin{tabular}{|l|l|l|l|}
0 & 0 & 1 & 1 \\
\hline 1 & & & 0 \\
\hline 1 & 1 & 0 \\
\hline
\end{tabular}

\section{Binary:01100111}

Figure 9: Example of the encoding method of novel WeberOrientation

Weber Orientation is quantized into 4 rangesIWBC-M and IWBC-O at each location are obtained.These feature maps are used to construct histograms which is used represent coral images

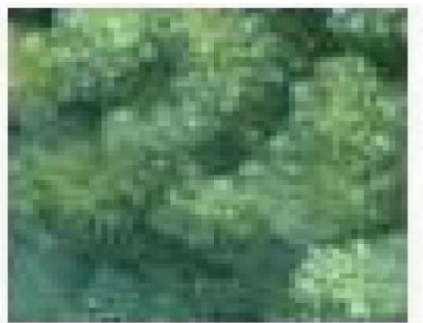

(a)

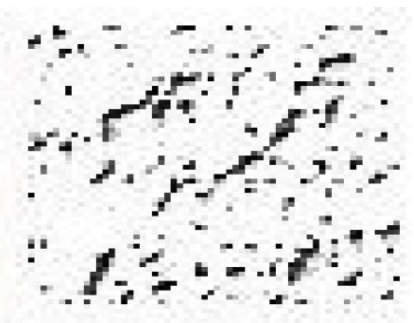

(b)
Figure 10:(a) Original coral image (b) Image after IWBC operation

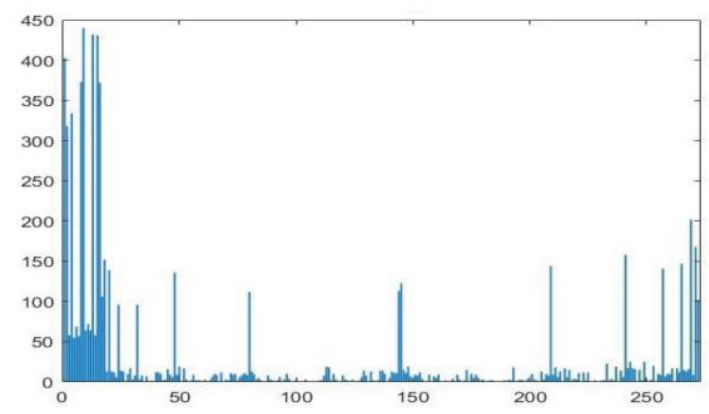

Figure 11: Histogram of IWBC operation with coral image

\section{E. Classifiers}

The Process of grouping test samples of texture in to classes refers to texture classification.Each classes are interrelated to some similarity criteria.The classification goal is to select appropriate category for an unidentified object,given a set of recognisedcategories.Sinceflawless classification is repeatedly unbearable, the classification may also be

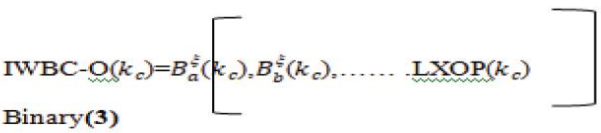

accomplished by determining the probability for each of known categories.

Two of the classifiers are used in the proposed system are:

- K NearestNeighbors,

- Random Forest.

\section{F. K-Nearest Neighbors}

K-Nearest Neighbor is one of the most essential classified algorithms in machine learning.It belongs to supervised learning.It is implemented in many application such as data mining,patternrecognition,andintrusion. The main purpose of $\mathrm{KNN}$ is to use the database and to classify new sample point from the datapoints of several classes based on similarity measure.(ex.distance function).

\section{G. Random Forest}

Random forest is also well-known as Random decision forest.It is a popular prognostic models for both grouping and deteriorationproblems. These method use numerous learning models to gain better analyticalresults. This model generates a random uncorrelated decision trees to arrive at the best possible result.

\section{EXPERIMENTAL RESULTS AND ANALYSIS}

\section{A. Dataset}

The Dataset which are used in the proposed system are EILAT. It contains 1123 image patches of size 64 taken from coral reef near Eilat in the Red sea.The image patches are pieces of longer images. With the same camera original images are taken under equivalent condition. The labels which are used here does not determine the coral species names.Classes of Eilat are listed below. Patches have been classified in to eight classes they areSand,Urchin,Branches Type I, Brain coral,Favidcoral,Branches Type II ,Dead coral and Branches Type III.Some sample images are listed below in Figure 12.

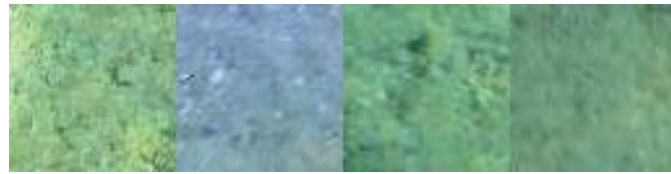

Figure 12: Sand 


\section{Coral Reef Image Classifications with Hybrid Methods}

Figure 13: Urchin

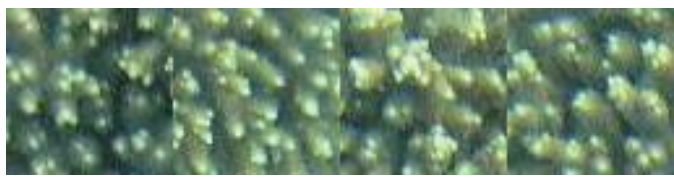

Figure 14: Branches Type I

Figure 15: Brain Coral

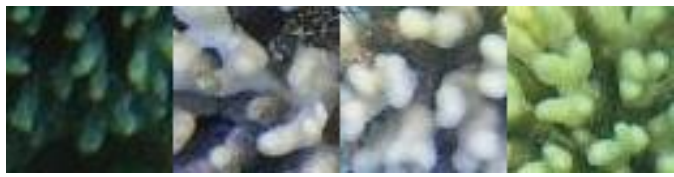

Figure 16: Branches Type II

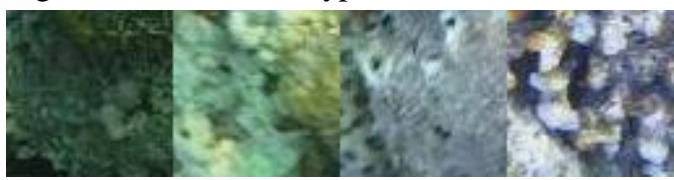

Figure 17: Dead Coral

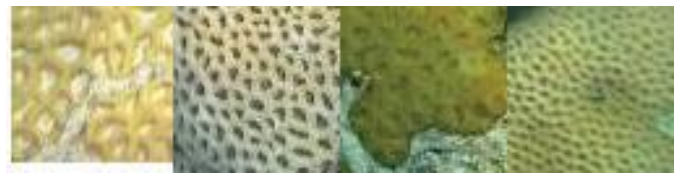

Figure 18: Favid Coral

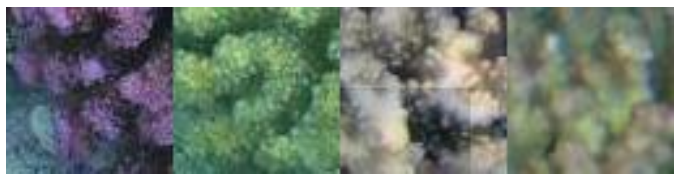

Figure 19: Branches Type III

\section{B. Performance Metrics}

Performance metrics are used to evaluate the classification models used in the proposed system. Experiments are conducted with the dataset Eilat.Matlab software is used in this proposed work for implementation. Some of the performance metrics which are used in this proposed work are accuracy, Sensitivity, Specificity,Precision,F1Score and Recall.The measures used to evaluate the above performance are listed below.

- True Positive(TP):Observation result is positive and the predicted result is positive

- False Negative(FN):Observation result is positive but the predicted result is negative.

- True Negative(TN): Observation result is negative and the predicted result is negative.

- False Positive(FP): Observation result is negative but the predicted result is positive.

\section{Accuracy}

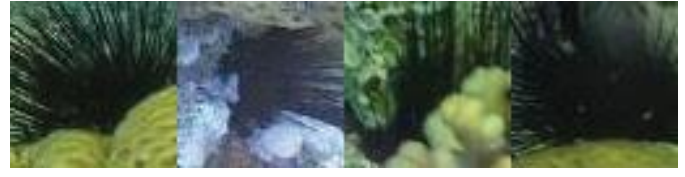

Accuracy is defined as how close a measured value is to the actual(true) value.It is the ratio of the number of correct

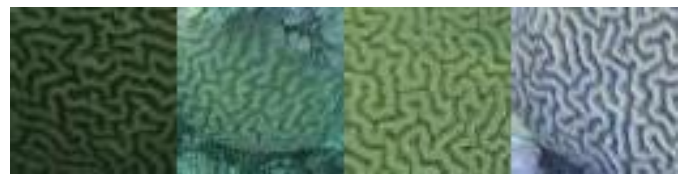

predictions to the total number of input samples.

Accuracy $=$ Number of Correct Predictions/Total number of Predictions

(4)

\section{Sensitivity (True Positive Rate)}

True Positive Rate relates to the fraction of positive data points that are correctly considered as positive with respect to all positive data points.

Sensitivity=TruePositive/False Negative+True Positive (5) Specificity (False Positive Rate)

False Positive Rate corresponds to the fraction of negative data points that are fallaciously considered as positive, with respect to all negative data points.

Specificity $=$ False Positive $/$ False Positive+True Negative(6)

\section{F1 Score}

F1 score is used to measure test accurateness. It is the Mean between precision and recall.

Mathematically it is represented as:

F1 Score $=(2 *$ Precision $*$ Recall $) /($ Precision + Recall $)$

\section{Precision}

It is the number of correct positive results divided by the number of positive results predicted by the classifier.

Precision $=$ True Positives $(\mathrm{TP}) /$ True Positives $(\mathrm{TP})+$ False Positives(FP)(8)

\section{Classification results}

The Experimental results are listed in table1 which shows the classification summary.Thefirst three rows of table 1 represents the results of coral classification using the handcrafted features such as Local Binary Pattern (LBP), Local Arc Pattern (LAP), and Webers Binary code (IWBC) separately. These handcrafted features are classified using the classifiers KNN and Random forest. By comparing the accuracy value of handcrafted features IWBC has high accuracy value with 97.4\%.Similarly the Sensitivity,

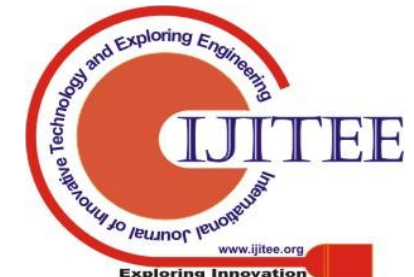




\begin{tabular}{|l|l|l|l|l|l|l|l|l|}
\hline Methods & \multicolumn{2}{|l|}{ Accuracy } & \multicolumn{2}{|l}{ Specificity } & \multicolumn{2}{l|}{ Sensitivity } & \multicolumn{2}{l|}{ Precision } \\
\hline classifier & KNN & RF & KNN & RF & KNN & RF & KNN & RF \\
\hline LBP & 93.2 & 93.3 & 95.9 & 95.9 & 77.3 & 64.2 & 76.0 & 82.2 \\
\hline LAP & 91.1 & 92.8 & 94.5 & 95.3 & 69.8 & 60.0 & 64.8 & 75.9 \\
\hline IWBC & 97.4 & 94.2 & 98.4 & 96.3 & 88.7 & 63.4 & 87.0 & 88.5 \\
\hline LBP+CNN & 95.77 & 95.4 & 95.7 & 97.7 & 85.7 & 82.5 & 81.3 & 88.4 \\
\hline LAP+CNN & 92.5 & 95.4 & 95.4 & 97.1 & 72.9 & 73.6 & 70.1 & 85.0 \\
\hline IWBC+CNN & $\mathbf{9 7 . 4}$ & $\mathbf{9 6 . 0}$ & 98.4 & 97.4 & 89.9 & 76.5 & 88.3 & 89.8 \\
\hline
\end{tabular}

Precision, Sensitivity and Specificity is also high compared to LAP and LBP with values $97.4 \%, 98.4 \%, 88.7 \%, 98.4 \%$ using KNN. Similarly the accuracy,precision,specificity of IWBC is very high using random forest with values 94.2\%,98.3\%, and96.3\%.Among the classification summary results of these handcrafted features using $\mathrm{KNN}$ and Random Forest as classifiers KNN score better performance than Random Forest.The Experimental results of handcrafted features combined with deep features also listed in last three rows of table 1.By comparing LBP + CNN,LAP $+\mathrm{CNN}, \mathrm{IWBC}+\mathrm{CNN}$ the accuracy value of IWBC + CNN acquires high value with 97.4\%.and also the Precision,Sensitivity,Specificity value is also very high compared to $\mathrm{LBP}+\mathrm{CNN}$ and $\mathrm{LAP}+\mathrm{CNN}$ with values $88.3 \%, 89.9 \%, 98.4 \%, 89.9 \%$ for $\mathrm{KNN}$ and 89.8\%,76.5\%,97.4\%using Random Forest..The Combined features performed better.The accuracy values of IWBC and IWBC with CNN have same value with $97.4 \%$ with $\mathrm{KNN}$ classifier and with random Forest IWBC score $94.2 \%$ and with IWBC $+\mathrm{CNN}$ it acquire $96.0 \%$ which is very high than other two methods.Among all these experimental results IWBC embedded with CNN acquire better results with KNN and Random Forest.

TABLE 1: Classified results of different feature representations

\begin{tabular}{|l|r|r|}
\hline LAP & 0.6643 & 0.6377 \\
\hline LAP+CNN & 0.7105 & 0.775 \\
\hline IWBC & 0.8758 & 0.6477 \\
\hline IWBC+CNN & 0.8865 & 0.8045 \\
\hline
\end{tabular}

Table 2 :F1 Score result of KNN and RFGRAPH 1: Comparision of $\mathrm{F} 1$ score results with $\mathrm{KNN}$ and $\mathrm{RF}$

\begin{tabular}{|l|r|l|}
\hline METHOD & KNN & RF \\
\hline LBP & 0.7597 & 0.6736 \\
\hline LBP+CNN & 0.8301 & 0.8499 \\
\hline
\end{tabular}

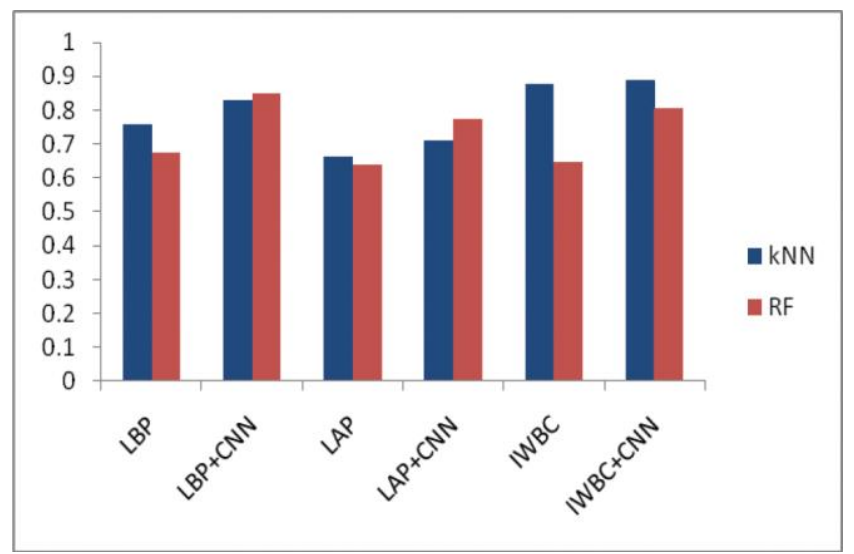

The Table 2 list the F1 Score result of KNN and Random Forest with hybrid methods of traditional and deep features.By comparing the results,IWBC with $\mathrm{CNN}$ acquire high F1 score value with $88.6 \%$ compared to Random Forest with $80.4 \%$.

The graph is also constructed for all methods such as LBP $\mathrm{LBP}+\mathrm{CNN}, \mathrm{LAP}, \mathrm{LAP}+\mathrm{CNN}$,IWBC,IWBC+CNN.Among all these methods IWBC with $\mathrm{CNN}$ shows better performance with KNN classifier. 


\section{Coral Reef Image Classifications with Hybrid Methods}

\section{RESULT AND CONCLUSION}

This paper proposed the state of art methods for extracting and classifying the features of coral reef images. Three traditional methods of Local Descriptors are reviewed and implemented. Later Experiments are conducted with the combination of deep features with these hand crafted features.KNN and Random Forest are used as classifiers. By

Comparing all the results shows that IWBC gives better accuracy value than other methods.IWBC is an effective, discriminative and robust feature descriptor which extract the feature of coral very accurately than other handcrafted features. When it combined with CNN its performance is effective with 97.4\%.These Experiments achieved state of art classification accuracy on EILAT dataset. Improved Weber Binary Code embedded with CNN are used for further research.

\section{REFERENCES}

1) Hughes TP, Baird AH, "climate change, human impacts, and the resilience of coral reefs", Science.2003 Aug 15.

2) Hoegh-Guldberg O, MumbyPJ."Coral reefs under rapid climate change and ocean acidification". Science 2007

3) M.S.A Marcos, Caesar, Soriano, and .Saloma, "Classification of coral reef images from underwater video using neural networks,"15 October 2005.

4) Paul Anton Letnes, Ingrid Myrnes ansen1,’Underwater hyper spectral classification of deepseacorals exposed to a toxic compound "Jun 14, 2017

5) Mehta.A.Ribeiro.E,"Coral Reef Texture Classification using support Vector Machines"In Proceedings of International Joint Conference on computer vision, Imaging and computer Graphics Theory and Applications, Barcelona, spain,8-11 March 2007

6) O.Pizarro, P.Rigby,'Towards image-based marine habitat classification", InProc OCEANS, 2008, pp 1-7

7) M.D.stokes and G.B.Deane,"Automated processing of coral reef benthic images" Limnol, Oceanography, 2009.

8) Z.Guo, L.Zhang, D, Zhang "A completed modelling of local binary pattern operator for texture classification" , IEEE Trans, Image Process.(9).(16).2010.1657-1663.

9) S.M.Shihavuddin1, NunoGracias"Image- based Coral ReefClassification and Thematic Mapping'RemoteSensing 5,(2013)1809-1841

10) NurhalisWahidina.b, VincentiusP.”Object based image analysis for coral reef benthic habitat mapping with several classification algorithms ProcediaEnvironmental sciences 24(2015).

11) Mohamed Essayed Elawady,"Sparse coral classification using Deep Convolutional Neural Networks", 29 Nov 2015

12) Mahmood," Coral classification with hybrid feature representations, "in Proc IEEE Int.Conf.Image Process 2016, pp519-523.

13) Mahmood A, Bennamoun $M$ “Automatic annotation of coral reefs using deep learning. In OCEANS 2016 Sep 20.

14) Jean-Nicola Blanchet, Sebastian Dery,kate Osborne "Automated annotation of corals in natural scene images using multiple texture representations",PeerJ Preprints,5 May 2016

15) YANG Guoqiang" High Resolution remote sensing classification of coral reef substrate, base on SVM-taken xisha an example"2016.

16) Mahmood, M.Bennamoun "RESFEATS: RESIDUAL NETWOK BASED FEATURES FOR IMAGE CLASSIFICATION"2017.

17) N. Ani Brown Mary,Dejey Dharma "Coral reef image classification employing Improved LDP for feature Extraction" Journal of visual communication and image representation,2017

18) InigoAlonso,"Coral-Segmentation: Training Dense Labeling Models with Sparse Ground Truth"2017 International Conference on Computer Vision Workshop(ICCVW).

19) LianXu, Mohammed Bennamoun "classification of corals in Reflectance and fluorescence Images Using convolutional Neural Network Representations" ICASSP 2018.Dianpeng Su,Fanlin Yang,
"Classification of Coral Reefs in the South china Sea by combining Airborne LiDAR Bathymetry bottom Waveforms and Bathymetric features" IEEE Transactions on Geoscience and Remote Sensing 2018.

20) Anabel, Gomez-Rios "Towards Highly Accurate Coral Texture Images Classification Using Deep Convolutional

21) Neural Networks and Data Augmentation" Expert System with Applications 2018.

22) Shinya Odagawa "Bottom-Type Classification in Coral Reef Area using Hyper spectral Bottom Index Imagery”IEEE 2017.

23) Ammar Mahmood" Deep Image Representations for Coral Image Classification"IEEE journal of oceanic engineering 2018

24) N.Ani Brown Mary "Classification of coral reef submarine images and Videos using a Novel $Z$ with Tilted $Z$ Local Binary Pattern(ZTZLBP)"Springer 2017

25) Bao-Qing Yang,"A novel face recognition method based on IWLD and IWBC",Springer + Business Media New York 2015
Published By:

Blue Eyes Intelligence Engineering \& Sciences Publication 